\title{
OCORRÊNCIA DE PESSOAS INFECTADAS PELO HIV QUE REALIZAM TRATAMENTO COM ANTIRRETROVIRAL EM UMA CIDADE DO SUL DO BRASIL: UM DESAFIO AOS PROFISSIONAIS DE SAÚDE
}

\author{
ADHERENCE OF HIV-INFECTED INDIVIDUALS TO \\ ANTIRETROVIRAL THERAPY IN A MUNICIPALITY IN \\ SOUTHERN BRAZIL: A CHALLENGE FOR HEALTH \\ PROFESSIONALS
}

Clin Biomed Res. 2019;39(2):140-143

1 Laboratório de Microbiologia, Faculdade de Biomedicina, Universidade do Planalto Catarinense (UNIPLAC). Lages, SC, Brasil.

2 Faculdade de Biomedicina, Universidade do Planalto Catarinense (UNIPLAC). Lages, SC, Brasil.

Autor correspondente: Rosiléia Marinho de Quadros biomedvety@gmail.com Universidade do Planalto Catarinense (UNIPLAC)

Av. Castelo Branco, 170. 88509-900, Lages, SC, Brasil.

\section{RESUMO}

Introdução: A utilização da terapia com antirretroviral (TARV) é um dos maiores desafios para o sucesso do tratamento para HIVIAIDS, uma vez que é uma das ferramentas contra a pandemia, possibilitando a melhoria da qualidade de vida e longevidade da população infectada. O objetivo deste trabalho foi verificar a ocorrência de pessoas infectadas com o HIV que faziam tratamento com TARV.

Métodos: O estudo foi realizado para verificar a ocorrência de pessoas infectadas com o HIV notificadas entre os anos de 2006 a 2016 que faziam tratamento com TARV, através das informações do Sistema Nacional de Informação de Agravos de Notificação (SINAN).

Resultados: Dos 715 pacientes notificados desde o ano de 2006, 597 (83,5\%) faziam o uso de TARV e 118 (16,5\%) abandonaram a terapia. Com relação ao perfil dos infectados, o maior número de notificações de HIV eram do sexo masculino $(54,83 \%)$, com idades entre 25 a 29 anos (56\%) dos casos.

Conclusão: Os dados apresentados no estudo mostram que algumas pessoas infectadas com HIV não dão continuidade ao tratamento, isso gera preocupação uma vez que mascara a realidade da infecção pelo vírus e diante deste cenário deve ocorrer o incentivo dos órgãos competentes para que registre os casos ocorrentes. Os profissionais de saúde nos seus mais diversos segmentos devem incentivar a utilização do TARV e notificar e acompanhar as pessoas que não retiram e não utilizam a medicação, e que sejam mais direcionadas as medidas de prevenção.

Palavras-chave: Vírus; AIDS; desafio; antirretroviral

\section{ABSTRACT}

Introduction: Adherence to antiretroviral therapy (ART) is a major challenge to the success of HIVIAIDS treatment. It is a weapon against the pandemic, being capable of improving the quality of life and longevity of the infected population. The aim of this study was to ascertain the adherence to ART in HIV-infected individuals.

Methods: The study assessed whether HIV-infected individuals with infection reported between 2006 and 2016 were receiving ART, using information from the National Information System for Notifiable Diseases (SINAN). 
Results: Of a sample of 715 patients, 597 (83.5\%) were using ART and 118 (16.5\%) discontinued treatment. Regarding the profile of HIV-infected individuals, most were male $(54.83 \%)$, aged 25 to 29 years $(56 \%)$.

Conclusions: These data show that a significant proportion of HIV-infected individuals discontinue treatment, which causes concern because it conceals the reality of HIV infection. Thus, authorities should call for proper reporting. Health professionals from different areas should encourage the use of ART and follow individuals who fail to adhere to treatment, while national health policies should focus on prevention.

Keywords: Vírus; Acquired Immunodeficiency Syndrome; challenge; antiretroviral therapy

Ainfecção pelo Vírus da Imunodeficiência Humana (HIV) durante a trajetória desde o seu descobrimento em diversas sociedades, apresentou características culturais de preconceito e discriminação, o indivíduo infectado passou a ser considerado a própria doença1.

A assistência à saúde dos indivíduos infectados pelo HIV no Brasil em decorrência da epidemia da AIDS (Síndrome da Imunodeficiência Adquirida) é um desafio. Esse desafio exige respostas por parte dos profissionais que além dos aspectos clínicos, também devem considerar os impactos sociais, psicológicos e econômicos que são associados aos estigmas sobre a infecção².

A incidência do HIVIAIDS parece estar relacionada com a exclusão social, onde grupos minoritários como homossexuais, os usuários de drogas e aqueles que sofrem com os efeitos da pobreza, são os mais suscetíveis ${ }^{3}$.

$O$ acesso gratuito e universal aos antirretrovirais (ARV) e ao teste anti-HIV no Brasil têm reduzido a morbidade e a mortalidade por AIDS, essa redução é decorrente também do esforço ao combate da discriminação dos pacientes positivos ${ }^{4}$.

Desde a metade dos anos 80 , antirretrovirais têm sido utilizados na AIDS. Os primeiros permitiam benefícios temporários, uma vez que apresentavam baixa eficácia na recuperação da capacidade imunológica e os efeitos limitados sobre a redução da carga viral ${ }^{5}$. O Brasil foi um dos primeiros países em desenvolvimento a garantir o acesso universal e gratuito à terapia antirretroviral (TARV) no Sistema Único de Saúde (SUS). A assistência as pessoas que vivem com HIV inclui programas que visam à redução das internações hospitalares, além da assistência ambulatorial especializada e a assistência domiciliar terapêutica ${ }^{6}$.

Diversas causas para a não adesão ao tratamento dos infectados com o vírus são citadas como, por exemplo, o modo de vida, principalmente as de baixas condições socioeconômicas, os efeitos do tratamento, o acesso limitado das populações marginalizadas, a falta de intervenções e informações eficazes para ajudar os pacientes a buscar as terapias necessárias para controlar a infecção?

A utilização do TARV é um dos maiores desafios para o sucesso do tratamento para HIVIAIDS, uma vez que tem produzido resultados positivos ao longo dos anos contra esta infecção e é uma das ferramentas contra esta pandemia, possibilitando a melhoria da qualidade de vida e longevidade da população infectada ${ }^{8}$. O estudo teve por objetivo verificar a ocorrência de pessoas notificadas infectadas com o HIV que faziam tratamento com TARV, através dos dados de notificação de doenças e agravos à saúde.

\section{MÉTODOS}

Foram utilizados para o estudo dados de pessoas notificadas com HIV que faziam a retirada do TARV para utilizar no seu tratamento, disponibilizados através do SINAN (Sistema Nacional de Informação de Agravos de Notificação) entre os anos de 2006 a 2016 na cidade de Lages, Santa Catarina, sul do Brasil. A pesquisa teve aprovação pelo Comitê de Ética em Pesquisa pela Universidade do Planalto Catarinense (UNIPLAC) parecer $n^{\circ}$ 1.790.999.

RESULTADOS

Conforme dados do SINAN, observou-se que o total de 715 pacientes notificados desde o ano de 2006 na cidade de Lages, 597 (83,5\%) faziam o uso de TARV, sendo que os outros $118(16,5 \%)$ não continuaram com a terapia, destes do sexo masculino foi de $(54,83 \%)$, e no sexo feminino $(45,17 \%)$, em relação a idade a maior ocorrência foi entre 25 a 29 anos (56\%).

\section{DISCUSSÃO}

A pesquisa revelou que grande parte das pessoas infectadas notificadas que não utilizavam, ou utilizavam inadequadamente o TARV eram do 
sexo masculino, esse dado também foi demonstrado em estudo transversal com pacientes portadores do HIV em um centro de atendimento especializado de saúde em Tubarão, Santa Catarina. Nessa cidade, $57 \%$ dos casos eram do sexo masculino e a forma de transmissão sexual foi citada como a causa da infecção ${ }^{9}$; também no nordeste do Brasil, a ocorrência de HIV no sexo masculino foi predominante, enfatizando que o diagnóstico no homem tem acontecido tardiamente e na faixa de idade de 30 a $34 \operatorname{anos}^{10}$. A maior notificação em relação à faixa etária gera preocupação uma vez que acomete a população jovem e com vida sexual ativa. A prevalência da infecção em adultos jovens está associada às práticas sexuais com múltiplos parceiros, uso incorreto ou o não uso do preservativo ${ }^{11}$.

As estratégias de políticas de saúde preventiva tem sido significativas no combate à transmissão do HIV, enfatizando as campanhas para uso de preservativos, a redução da transmissão vertical, o controle de qualidade nos bancos de sangue e a distribuição gratuita de antirretrovirais ${ }^{12}$. Outra cidade no sul do Brasil em um estudo para verificar o uso de TARV, 476 pacientes com HIV que foram notificados, $35,9 \%$ deles não realizaram tratamento, número maior de resistência e abandono ao tratamento quando comparado com o presente estudo na cidade de Lages ${ }^{9}$.

Os motivos que levam os pacientes a não darem continuidade ao tratamento com o TARV, dentre eles destacam-se a ausência de manifestações sintomáticas que o vírus do HIV causa, os efeitos indesejáveis que o tratamento com a medicação causa, como mal estar, náuseas, além de oscilações psicológicas como estresse e irritabilidade. Outro fator a ser também considerado é principalmente a não aceitação da doença ${ }^{13}$. Cabe ressaltar que o desamparo familiar e problemas econômicos também são fatores importantes a serem citados para o abandono da terapia ${ }^{14}$.

A não adesão à terapêutica antirretroviral é um dos perigos mais ameaçadores para o sucesso do programa de prevenção a nível individual, o que pode levar por consequência a disseminação do vírus em termos coletivo ${ }^{15}$.

Com a disponibilidade de TARV, o HIV tornou-se uma infecção crônica que requer gestão por parte dos órgãos de saúde ao longo do tempo ${ }^{16}$. Dada a esta situação a prevenção é fundamental, e estratégias devem ser reavaliadas por parte da saúde pública. Nos Estados Unidos os recursos federais para HIVIAIDS somam $3 \%$ do orçamento para a prevenção, enquanto $61 \%$ são gastos para os cuidados e tratamento ${ }^{17}$.

A resposta dos serviços em saúde contribuiu ao longo dos anos para o conhecimento do crescimento ou redução dos casos de HIVIAIDS em várias regiões do Brasil. Estes dados podem demonstrar que pacientes com a enfermidade acessam tardiamente aos serviços de saúde e assim podem ter benefícios limitados da terapia específica ${ }^{6}$.

As doenças de notificação compulsória são importantes para apontar enfermidades a nível mundial ou de esferas nacionais de elevada gravidade e magnitude, por consequência são capazes de levar a disseminação do agente causal e desenvolver quadros de surtos ou epidemias que exigem dos sistemas de vigilância em saúde o monitoramento adequado de medidas eficazes de prevenção e controle destas doenças ${ }^{18}$.

Os dados apresentados no estudo mostram que existem pessoas infectadas com HIV que não continuam com o tratamento com TARV, isso gera preocupação uma vez que mascara a realidade da infecção pelo vírus e a continuidade ao tratamento, e diante deste cenário deve ocorrer o incentivo dos órgãos competentes para que registrem os casos ocorrentes, de pacientes que não prosseguem com o tratamento, já que as políticas públicas de saúde estão direcionadas as enfermidades regionais e a sua prevenção e controle. Em relação à utilização do TARV pelos infectados mesmo que tenha sido considerada boa, pelo grande número de pessoas que estão sendo tratadas é importante lembrar que a terapia é dada de forma gratuita e que estes dados devem ser acompanhados a cada ano para que cada vez menos ocorra desistência. Embora muitas ações em saúde ainda sejam necessárias para diminuir a epidemia de AIDS, será impossível pôr fim à epidemia sem disponibilizar o tratamento do HIV para todos que precisam, a meta nacional é que até $2020,90 \%$ de todas as pessoas com infecção pelo HIV diagnosticada receberão terapia antirretroviral ininterruptamente ${ }^{19}$.

Cabe ressaltar também que a adesão é um ato de caráter dinâmico a ser constantemente estimulado ${ }^{20}$, e que os profissionais de saúde nos seus mais diversos segmentos devem seguir encorajando os infectados com HIV a aderir e seguir com a terapia antirretroviral, uma aproximação com o paciente que em conjunto com seus familiares contribuirão para o sucesso da terapia.

\section{Conflitos de Interesse}

Os autores declaram não ter conflitos de interesse. 


\section{REFERÊNCIAS}

1. Schaurich D, Coelho DF, Motta MGC A cronicidade no processo saúdedoença: repensando à epidemia da AIDS após os anti-retrovirais. $R$ Enferm. 2006;14:455-62.

2. Nobre AQTC, Costa IS, Bernardes KO. A fisioterapia no contexto do HIV/ AIDS. Fisioter Mov. 2008;21:11-8.

3. Silva CRC. A amizade e a politização de redes sociais de suporte: reflexões com base em estudo de ONG/AIDS na grande São Paulo. Saude Soc. 2009;18(4):721-32. http://dx.doi.org/10.1590/S010412902009000400015.

4. Paiva V, Pupo LR, Barboza R. O direito à prevenção e os desafios da redução da vulnerabilidade ao HIV no Brasil. Rev Saude Publica. 2006;40(Supl):10919. http://dx.doi.org/10.1590/ S0034-89102006000800015. PMid:16729166.

5. Seidl EMF, Melchiades A, Farias V, Brito A. Pessoas vivendo com HIVI AIDS: variáveis associadas à adesão ao tratamento anti-retroviral. Rev Saude Publica. 2007;23(10):2305-16. http://dx.doi.org/10.1590/S0102311X2007001000006.

6. Dourado I, Veras MASM, Barreira D, Brito AM. Tendências da epidemia de AIDS no Brasil após a terapia anti-retroviral. Rev Saude Publica. 2006;40(Supl):917. http://dx.doi.org/10.1590/ S0034-89102006000800003. PMid:16729154.

7. Bonolo PF, Gomes RRFM, Guimarães MDC. Adesão à terapia anti-retroviral (HIV/aids):fatores associados e medidas da adesão. Epidemiol Serv Saude. 2007;16:261-78.

8. Gir E, Vaichulonis CG, Oliveira MD. Adesão à terapêutica antirretroviral por indivíduos com HIVIAids assistidos em uma instituição do interior paulista. Rev Latino-Am Enf. 2005;13(5):634-41. http:// dx.doi.org/10.1590/S010411692005000500005.

9. Schuelter-Trevisol F, Paolla P Justino AZ, Pucci N, Silva ACB. Perfil epidemiológico dos pacientes com HIV atendidos no sul do Estado de Santa Catarina, Brasil, em 2010. Epidemiol Serv Saude. 2013;22(1):8794. http://dx.doi.org/10.5123/S167949742013000100009.

10. Pedrosa NL, Paiva SS, Almeida RLF, Holanda ER, Kerr LRFS, Galvão MTG. Série histórica da AIDS no Estado do Ceará, Brasil. Cien Saude Colet. 2015;20(4):1177-84. http://dx.doi.org/10.1590/141381232015204.00582014. PMid:25923628.

11. Dessunti E, Mathias R, Advincula AO. Fatores psicossociais e comportamentais associados ao risco de DST/AIDS entre estudantes da área de saúde. Rev Latino-Am Enf. 2007;15(2):267-74. http://dx.doi.org/10.1590/S010411692007000200012.

12. Reis CB, Bernardes EB. O que acontece atrás das grades: estratégias de prevenção desenvolvidas nas delegacias civis contra HIVIAIDS e outras doenças sexualmente transmissíveis. Cien Saude Colet. 2011;16(7):33318. http://dx.doi.org/10.1590/ S1413-81232011000800032. PMid:21808920.

13. Galano E, Turato ER, Delmas P, Côté J, Gouvea AFTB, Succi RCM, et al. Vivências dos adolescentes soropositivos para HIVIAIDS: estudo qualitativo. Rev Paul Pediatr. 2016;34(2):171-7. http://dx.doi. org/10.1016/j.rpped.2015.10.004. PMid:26611887.
14. Santos FB, Seidl EMF. Caracterização de pessoas com HIV/aids em abandono do tratamento antirretroviral e a busca consentida de casos. Brasília Medica. 2011;48:268-76.

15. Colombrini MRC, Lopes MHBM, Figueiredo RM. Adesão à terapia antirretroviral para HIVIAIDS. Rev Esc Enferm USP. 2006;40(4):57681. http://dx.doi.org/10.1590/ S0080-62342006000400018. PMid:17310576.

16. Kendall CE, Shoemaker ES, Crowe L, MacPherson P, Becker ML, Levreault $E$, et al. Patient activation among people living with HIV: a cross-section comparative analisys with people living with diabetes mellitus. AIDS Care. 2018;30(11):1444-51. http:// dx.doi.org/10.1080/09540121.2018.14 69723. PMid:29792355.

17. Eweka I, Fleuriscar J, Fleuriscar J, Adeyemi-Jones A, Dillon B, Albritton T. Medical students reflect on the future of Pre-Exposure Prophylaxis use among adolescents and young adults. Prev Med. 2018;113:1223. http://dx.doi.org/10.1016/j. ypmed.2018.05.020. PMid:29792894.

18. Silva GA, Oliveira CMG. O registro das doenças de notificação compulsória: a participação dos profissionais da saúde e da comunidade. Rev Epidemiol Control Infect. 2014;4(3):215-20. http://dx.doi. org/10.17058/reci.v4i3.4578.

19. Programa Conjunto das Nações Unidas sobre HIVIAIDS - UNAIDS. 90-90-90: uma meta ambiciosa de tratamento para contribuir para o fim da epidemia de AIDS. Genebra: UNAIDS; 2015.

20. Chesney MA, Morin M, Sherr L. Adherence to HIV combination therapy. Soc Sci Med. 2000;50(11):1599-605. http://dx.doi. org/10.1016/S0277-9536(99)00468-2. PMid:10795966.

Recebido: 25 ago, 2018 Aceito: 6 maio, 2019 